\title{
Thermodynamic Investigation of 1,3,5-Trioxane, Methyl Acrylate, Methyl Acetate, and Water Mixtures, in Terms of NRTL and UNIQUAC Models
}

\author{
Hui Zhao, ${ }^{\dagger, \ddagger} \mathrm{Jie} \mathrm{Li,}{ }^{\ddagger}$ Lei Wang, ${ }^{\ddagger}$, Chunshan Li, ${ }^{*, \neq, \S}$ and Ping $\mathrm{Li}^{\prime}$ \\ ${ }^{\dagger}$ University of Chinese Academy of Sciences, Beijing 100049, China \\ ‡State Key Laboratory of Multiphase Complex Systems, Beijing Key Laboratory of Ionic Liquids Clean \\ Process, Zhongke Langfang Institute of Process Engineering, Institute of Process Engineering, Chinese \\ Academy of Sciences, Beijing 100190, China \\ $\S$ Innovation Academy for Green Manufacture, Chinese Academy of Sciences, Beijing 100190, China \\ "State Key Laboratory of High-efficiency Utilization of Coal and Green Chemical Engineering, School of \\ Chemistry and Chemical Engineering, Ningxia University, Yinchuan, 750021, People's Republic of China
}

Part 1. Data for SLE system.

Part 2. Data for VLE binary systems.

Part 3. Data for LLE ternary system.

Part 4. Data for LLE quaternary system.

Part 5. Information for separation process. 
Part 1. Data for SLE system.

Table S1. Solubility of 1,3,5-trioxane (solid) in MAc/MA at $101.3 \mathbf{k P a}^{\mathrm{a}}$.

$\begin{array}{cccccc}\text { Solvent } & T(\mathrm{~K}) & x & \text { Solvent } & T(\mathrm{~K}) & x \\ \text { MAc } & 325.95 & 0.8956 & \text { MA } & 325.85 & 0.8851 \\ & 320.55 & 0.8170 & & 318.85 & 0.7702 \\ & 311.65 & 0.7019 & & 312.05 & 0.6651 \\ & 304.05 & 0.6035 & & 304.45 & 0.5624 \\ & 295.85 & 0.5038 & & 297.05 & 0.4624\end{array}$

${ }^{\text {a }}$ The standard uncertainties are $u(P)=0.1 \mathrm{kPa}, u(T)=0.1 \mathrm{~K}, u(x$ in $M A c)=u(x$ in $M A)=0.0006$. 
Part 2. Data for VLE binary systems.

Table S2. Experimental and estimated VLE data for 1,3,5-Trioxane (1) + MAc (2) binary system at $101.3 \mathrm{kPa}^{\mathrm{a}}$.

$\begin{array}{ccccccccc}T^{\text {Exp }} & T_{N R T L}^{\text {Est }} & T_{U N I Q}^{\text {Est }} & x_{1}^{\text {Exp }} & x_{1 N}^{\text {Est }} & x_{1 U}^{\text {Est }} & y_{1}^{\text {Exp }} & y_{1 N}^{\text {Est }} & y_{1 U}^{\text {Est }} \\ 330.95 & 331.02 & 330.60 & 0.0276 & 0.0276 & 0.0276 & 0.0022 & 0.0021 & 0.0022 \\ 331.95 & 331.95 & 331.76 & 0.0696 & 0.0695 & 0.0695 & 0.0051 & 0.0060 & 0.0059 \\ 333.05 & 333.04 & 332.92 & 0.1068 & 0.1069 & 0.1070 & 0.0111 & 0.0104 & 0.0099 \\ 334.35 & 334.25 & 334.56 & 0.1587 & 0.1588 & 0.1588 & 0.0175 & 0.0168 & 0.0164 \\ 335.05 & 335.00 & 335.64 & 0.1912 & 0.1914 & 0.1914 & 0.0233 & 0.0211 & 0.0212 \\ 336.15 & 335.98 & 337.06 & 0.2333 & 0.2329 & 0.2324 & 0.0233 & 0.0268 & 0.0282 \\ 337.55 & 337.43 & 339.21 & 0.2895 & 0.2899 & 0.2888 & 0.0391 & 0.0355 & 0.0400 \\ 339.05 & 338.79 & 340.98 & 0.3357 & 0.3350 & 0.3328 & 0.0374 & 0.0435 & 0.0513\end{array}$

a The standard uncertainties are $u(P)=0.1 \mathrm{kPa}, u(T)=0.1 \mathrm{~K}, u(x)=0.0030, u(y)=0.0002$.

Table S3. Experimental and estimated VLE data for 1,3,5-Trioxane (1) + MA (2) binary system at $101.3 \mathrm{kPa}^{\mathrm{a}}$.

\begin{tabular}{ccccccccc}
$T^{\text {Exp }}$ & $T_{\text {NRTL }}^{\text {Est }}$ & $T_{U N I Q}^{\text {Est }}$ & $x_{1}^{\text {Exp }}$ & $x_{1 N}^{\text {Est }}$ & $x_{1 U}^{\text {Est }}$ & $y_{1}^{\text {Exp }}$ & $y_{1 N}^{\text {Est }}$ & $y_{1 U}^{\text {Est }}$ \\
\hline 355.05 & 355.51 & 355.57 & 0.0947 & 0.0947 & 0.0947 & 0.0266 & 0.0267 & 0.0253 \\
357.15 & 357.30 & 357.95 & 0.1885 & 0.1885 & 0.1882 & 0.0547 & 0.0546 & 0.0599 \\
359.45 & 359.31 & 360.43 & 0.2833 & 0.2834 & 0.2824 & 0.0900 & 0.0882 & 0.1054 \\
362.15 & 361.85 & 363.10 & 0.3840 & 0.3842 & 0.3825 & 0.1375 & 0.1346 & 0.1657 \\
364.85 & 364.90 & 365.72 & 0.4841 & 0.4833 & 0.4810 & 0.1831 & 0.1964 & 0.2367
\end{tabular}

a The standard uncertainties are $u(P)=0.1 \mathrm{kPa}, u(T)=0.1 \mathrm{~K}, u(x)=0.0014, u(y)=0.0003$. 
Part 3. Data for LLE ternary system.

Table S4. Experimental LLE data for 1,3,5-Trioxane (1) + MA (2) + Water (3) ternary system at $101.3 \mathrm{kPa}^{\mathrm{a}}$.

\begin{tabular}{|c|c|c|c|c|c|c|}
\hline & \multicolumn{3}{|c|}{ Oil phase $\left(\mathrm{mol} \cdot \mathrm{mol}^{-1}\right)$} & \multicolumn{3}{|c|}{ Water phase $\left(\mathrm{mol} \cdot \mathrm{mol}^{-1}\right)$} \\
\hline & $o_{1}$ & $\mathrm{O}_{2}$ & $\mathrm{O}_{3}$ & $w_{1}$ & $w_{2}$ & $w_{3}$ \\
\hline \multirow{7}{*}{$T=313.15 \mathrm{~K}$} & 0.0457 & 0.8041 & 0.1502 & 0.0047 & 0.014 & 0.9813 \\
\hline & 0.1051 & 0.7155 & 0.1794 & 0.0114 & 0.0121 & 0.9765 \\
\hline & 0.1687 & 0.6759 & 0.1554 & 0.0191 & 0.0147 & 0.9662 \\
\hline & 0.3042 & 0.5230 & 0.1728 & 0.0360 & 0.0126 & 0.9514 \\
\hline & 0.3217 & 0.4976 & 0.1807 & 0.0383 & 0.0118 & 0.9499 \\
\hline & 0.3446 & 0.4708 & 0.1846 & 0.0447 & 0.0131 & 0.9422 \\
\hline & 0.3895 & 0.3961 & 0.2144 & 0.0562 & 0.0159 & 0.9279 \\
\hline \multirow[t]{4}{*}{ standard uncertainty, $u$} & 0.0029 & 0.0065 & 0.0081 & 0.0013 & 0.0012 & 0.0022 \\
\hline & 0.0461 & 0.7964 & 0.1575 & 0.0071 & 0.0108 & 0.9821 \\
\hline & 0.1161 & 0.7192 & 0.1647 & 0.0133 & 0.0124 & 0.9743 \\
\hline & 0.1690 & 0.6611 & 0.1699 & 0.0188 & 0.0125 & 0.9687 \\
\hline \multirow[t]{4}{*}{$T=323.15 \mathrm{~K}$} & 0.2986 & 0.5039 & 0.1975 & 0.0404 & 0.0125 & 0.9471 \\
\hline & 0.3110 & 0.4896 & 0.1994 & 0.0412 & 0.0131 & 0.9457 \\
\hline & 0.3366 & 0.4602 & 0.2032 & 0.0476 & 0.0146 & 0.9378 \\
\hline & 0.3569 & 0.3558 & 0.2873 & 0.0622 & 0.0202 & 0.9176 \\
\hline standard uncertainty, $u$ & 0.0018 & 0.0052 & 0.0059 & 0.0005 & 0.0004 & 0.0007 \\
\hline
\end{tabular}

${ }^{\text {a }}$ For both conditions, the standard uncertainties for pressure and temperature are $u(P)=0.1 \mathrm{kPa}$ and $u(T)=0.1 \mathrm{~K}$.

Table S5. Estimated LLE data for 1,3,5-Trioxane (1) + MA (2) + Water (3) ternary system.

\begin{tabular}{|c|c|c|c|c|c|c|c|c|}
\hline & \multicolumn{5}{|c|}{ Oil phase $\left(\mathrm{mol} \cdot \mathrm{mol}^{-1}\right)$} & \multicolumn{3}{|c|}{ Water phase $\left(\mathrm{mol} \cdot \mathrm{mol}^{-1}\right)$} \\
\hline & $o_{1 N}$ & $o_{1 U}$ & $\mathrm{O}_{2 \mathrm{~N}}$ & $\mathrm{O}_{2 U}$ & $w_{1 N}$ & $w_{1 U}$ & $w_{2 N}$ & $w_{2 U}$ \\
\hline \multirow{7}{*}{$T=313.15 \mathrm{~K}$} & 0.0460 & 0.0458 & 0.7992 & 0.7934 & 0.0047 & 0.0047 & 0.0127 & 0.0131 \\
\hline & 0.1064 & 0.1063 & 0.7325 & 0.7297 & 0.0113 & 0.0113 & 0.0130 & 0.0133 \\
\hline & 0.1707 & 0.1703 & 0.6616 & 0.6633 & 0.0188 & 0.0189 & 0.0133 & 0.0133 \\
\hline & 0.2987 & 0.3016 & 0.5209 & 0.5319 & 0.0364 & 0.0365 & 0.0137 & 0.0133 \\
\hline & 0.3160 & 0.3213 & 0.5022 & 0.5139 & 0.0390 & 0.0390 & 0.0137 & 0.0132 \\
\hline & 0.3474 & 0.3497 & 0.4673 & 0.4842 & 0.0443 & 0.0449 & 0.0137 & 0.0134 \\
\hline & 0.4040 & 0.4045 & 0.4036 & 0.4269 & 0.0549 & 0.0576 & 0.0136 & 0.0138 \\
\hline \multirow{7}{*}{$T=323.15 \mathrm{~K}$} & 0.0513 & 0.0502 & 0.8014 & 0.8126 & 0.0064 & 0.0066 & 0.0108 & 0.0113 \\
\hline & 0.1111 & 0.1118 & 0.7233 & 0.7237 & 0.0140 & 0.0139 & 0.0117 & 0.0120 \\
\hline & 0.1559 & 0.1569 & 0.6646 & 0.6590 & 0.0200 & 0.0196 & 0.0124 & 0.0125 \\
\hline & 0.2823 & 0.2820 & 0.4989 & 0.4823 & 0.0405 & 0.0401 & 0.0144 & 0.0140 \\
\hline & 0.2906 & 0.2891 & 0.4882 & 0.4728 & 0.0421 & 0.0417 & 0.0145 & 0.0142 \\
\hline & 0.3200 & 0.3152 & 0.4499 & 0.4388 & 0.0482 & 0.0481 & 0.0150 & 0.0147 \\
\hline & 0.3819 & 0.3731 & 0.3666 & 0.3628 & 0.0641 & 0.0660 & 0.0161 & 0.0159 \\
\hline
\end{tabular}


Part 4. Data for LLE quaternary system.

Table S6. Estimated data for 1,3,5-Trioxane (1) + MA (2) + MAc (3) + Water (4) quaternary system by NRTL model.

\begin{tabular}{ccccccccc} 
& \multicolumn{4}{c}{ Oil phase $\left(\mathrm{mol} \cdot \mathrm{mol}^{-1}\right)$} & \multicolumn{3}{c}{ Water phase $\left(\mathrm{mol} \cdot \mathrm{mol}^{-1}\right)$} \\
\cline { 2 - 9 } & $o_{1 N}$ & $o_{2 N}$ & $o_{3 N}$ & $o_{4 N}$ & $w_{1 N}$ & $w_{2 N}$ & $w_{3 N}$ & $w_{4 N}$ \\
\hline \multirow{3}{*}{$=313.15 \mathbf{K}$} & 0.1517 & 0.2967 & 0.4463 & 0.1052 & 0.0164 & 0.0063 & 0.0225 & 0.9548 \\
& 0.1929 & 0.2458 & 0.3733 & 0.1880 & 0.0215 & 0.0066 & 0.0223 & 0.9495 \\
& 0.1970 & 0.2757 & 0.3595 & 0.1678 & 0.0213 & 0.0070 & 0.0206 & 0.9512 \\
& 0.2651 & 0.1624 & 0.2577 & 0.3148 & 0.0386 & 0.0070 & 0.0222 & 0.9323 \\
& 0.2689 & 0.1842 & 0.2560 & 0.2908 & 0.0369 & 0.0072 & 0.0208 & 0.9350 \\
& 0.2515 & 0.2019 & 0.2792 & 0.2673 & 0.0324 & 0.0072 & 0.0209 & 0.9396 \\
\hline \multirow{5}{*}{ 323.15 K } & 0.2479 & 0.1527 & 0.2361 & 0.3633 & 0.0417 & 0.0087 & 0.0247 & 0.9249 \\
& 0.2545 & 0.1714 & 0.2473 & 0.3268 & 0.0394 & 0.0087 & 0.0238 & 0.9281 \\
& 0.2424 & 0.1965 & 0.2663 & 0.2949 & 0.0338 & 0.0086 & 0.0226 & 0.9351 \\
& 0.2544 & 0.1347 & 0.2242 & 0.3868 & 0.0468 & 0.0088 & 0.0261 & 0.9184 \\
\hline
\end{tabular}

Table S7. Estimated data for 1,3,5-Trioxane (1) + MA (2) + MAc (3) + Water (4) quaternary system by UNIQUAC model.

\begin{tabular}{ccccccccc} 
& \multicolumn{4}{c}{ Oil phase $\left(\mathrm{mol} \cdot \mathrm{mol}^{-1}\right)$} & \multicolumn{3}{c}{ Water phase $\left(\mathrm{mol} \cdot \mathrm{mol}^{-1}\right)$} \\
\cline { 2 - 9 } & $o_{1 U}$ & $o_{2 U}$ & $o_{3 U}$ & $o_{4 U}$ & $w_{1 U}$ & $w_{2 U}$ & $w_{3 U}$ & $w_{4 U}$ \\
\hline \multirow{3}{*}{$=313.15 \mathbf{K}$} & 0.1504 & 0.2883 & 0.4361 & 0.1251 & 0.0162 & 0.0063 & 0.0218 & 0.9557 \\
& 0.1959 & 0.2454 & 0.3754 & 0.1832 & 0.0214 & 0.0065 & 0.0222 & 0.9499 \\
& 0.1959 & 0.2735 & 0.3521 & 0.1785 & 0.0210 & 0.0070 & 0.0205 & 0.9515 \\
& 0.2696 & 0.1657 & 0.2617 & 0.3031 & 0.0393 & 0.0070 & 0.0225 & 0.9312 \\
& 0.2676 & 0.1851 & 0.2583 & 0.2890 & 0.0373 & 0.0073 & 0.0212 & 0.9342 \\
& 0.2527 & 0.2038 & 0.2813 & 0.2622 & 0.0326 & 0.0072 & 0.0211 & 0.9391 \\
\hline \multirow{3}{*}{$=323.15 \mathbf{K}$} & 0.2498 & 0.1538 & 0.2348 & 0.3616 & 0.0417 & 0.0086 & 0.0242 & 0.9255 \\
& 0.2442 & 0.1673 & 0.2408 & 0.3477 & 0.0387 & 0.0087 & 0.0234 & 0.9292 \\
& 0.2301 & 0.1904 & 0.2596 & 0.3198 & 0.0333 & 0.0087 & 0.0227 & 0.9353 \\
& 0.2585 & 0.1348 & 0.2227 & 0.3840 & 0.0469 & 0.0085 & 0.0252 & 0.9194
\end{tabular}


Part 5. Information for separation process.

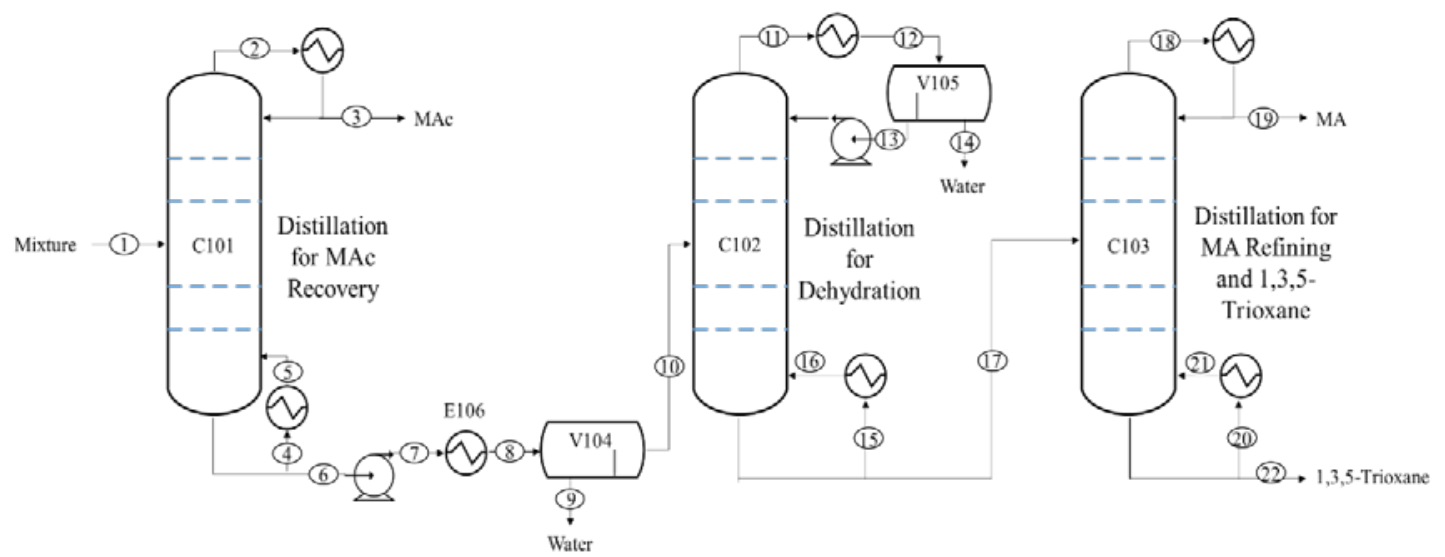

Figure S1. Separation flow scheme for 1,3,5-Trioxane + MA + MAc + Water quaternary system with stream number.

Table S8. Operational conditions of the distillation columns and decanters.

$\begin{array}{ccccccccc}\text { Column } & \text { Stages } & \begin{array}{c}\text { Feed } \\ \text { Stage }\end{array} & \begin{array}{c}\text { Reflux } \\ \text { Ratio }\end{array} & \begin{array}{c}\text { Distillate } \\ \text { Rate }(\mathrm{kg} / \mathrm{h})\end{array} & \begin{array}{c}\text { Bottoms } \\ \text { Rate }(\mathrm{kg} / \mathrm{h})\end{array} & \text { Decanter } & T(\mathrm{~K}) & \begin{array}{c}P \\ (\mathrm{kPa})\end{array} \\ \mathbf{C 1 0 1} & 42 & 21 & 3.025 & 860 & - & \mathbf{V 1 0 4} & 303.15 & 101.33 \\ \mathbf{C 1 0 2} & 8 & 3 & - & - & 120 & \mathbf{V 1 0 5} & 303.15 & 55 \\ \mathbf{C 1 0 3} & 30 & 5 & 2 & 70 & - & & & \end{array}$


Table S9. Streams information of separation process.

\begin{tabular}{ccccccccc} 
No. & $T(\mathrm{~K})$ & $\mathrm{P}(\mathrm{kPa})$ & Vapor Fraction & Mass Flow $(\mathrm{kg} / \mathrm{h})$ & MAc & MA & $1,3,5$-Trioxane & Water \\
\cline { 6 - 8 } 1 & 303.15 & 101.33 & 0.00 & 1000.00 & 0.855 & 0.075 & 0.055 & 0.015 \\
2 & 299.65 & 30.37 & 1.00 & 3461.50 & 0.994 & 0.005 & trace & 640 PPM \\
3 & 299.25 & 30.00 & 0.00 & 860.00 & 0.994 & 0.005 & trace & 640 PPM \\
4 & 331.45 & 44.63 & 0.00 & 1797.84 & 22 PPM & 0.704 & 0.153 & 0.143 \\
5 & 336.45 & 45.00 & 0.93 & 1797.84 & 22 PPM & 0.704 & 0.153 & 0.143 \\
6 & 336.45 & 45.00 & 0.00 & 140.00 & 7 PPM & 0.504 & 0.393 & 0.103 \\
7 & 336.55 & 200.00 & 0.00 & 140.00 & 7 PPM & 0.504 & 0.393 & 0.103 \\
8 & 293.15 & 101.33 & 0.00 & 140.00 & 7 PPM & 0.504 & 0.393 & 0.103 \\
9 & 303.15 & 101.33 & 0.00 & 19.54 & 31 PPM & 0.003 & 0.267 & 0.794 \\
10 & 303.15 & 101.33 & 0.00 & 120.40 & 3 PPM & 0.585 & 0.413 & 0.001 \\
11 & 339.05 & 61.43 & 1.00 & 410.41 & 0.007 & 0.992 & 998 PPM & 413 PPM \\
12 & 337.75 & 60.00 & 1.00 & 371.35 & 0.015 & 0.984 & 41 PPM & 562 PPM \\
13 & 303.15 & 55.00 & 0.00 & 370.95 & 0.015 & 0.985 & 41 PPM & 161 PPM \\
14 & 303.15 & 55.00 & 0.00 & 0.15 & 0.002 & 0.001 & trace & 0.149 \\
15 & 348.65 & 68.57 & 0.00 & 415.17 & 70 PPM & 0.769 & 0.231 & 43 PPB \\
16 & 354.05 & 70.00 & 0.71 & 415.17 & 70 PPM & 0.769 & 0.231 & 43 PPB \\
17 & 354.05 & 70.00 & 0.00 & 120.00 & 30 PPM & 0.585 & 0.415 & 10 PPB \\
18 & 318.65 & 20.35 & 1.00 & 169.69 & 50 PPM & 0.995 & 0.005 & 16 PPB \\
19 & 310.25 & 20.00 & 0.00 & 70.33 & 50 PPM & 0.995 & 0.005 & 17 PPB \\
20 & 351.85 & 29.66 & 0.00 & 230.01 & trace & 0.009 & 0.991 & Trace \\
21 & 352.25 & 30.00 & 0.78 & 230.01 & trace & 0.009 & 0.991 & Trace \\
22 & 352.25 & 30.00 & 0.00 & 49.67 & trace & 0.003 & 0.549 & Trace
\end{tabular}

